\title{
ISOLATION AND CHARACTERIZATION OF TWO MYOACTIVE NEUROPEPTIDES: FURTHER EVIDENCE OF AN INVERTEBRATE PEPTIDE FAMILY ${ }^{1}$
}

\author{
MICHAEL O'SHEA, ${ }^{2}$ JANE WITTEN, AND MARTIN SCHAFFER* \\ Department of Pharmacological and Physiological Sciences and * Department of Psychiatry, The University of Chicago, \\ Chicago, Illinois 60637
}

Received June 13, 1983; Revised September 16, 1983; Accepted September 19, 1983

\begin{abstract}
The neuropeptide proctolin acts as a neuromuscular co-transmitter in insect skeletal muscle. As a prelude to determining whether other peptides may function in a similar way, we are attempting to isolate and characterize the chemical nature of new myoactive neuropeptides in insects. We examined the corpus cardiacum, a major insect neurosecretory structure of the American cockroach (Periplaneta americana), using a skeletal muscle bioassay and high pressure liquid chromatography fractionation and identified two myoactive factors, MI and MII. They are synthesized in the corpus cardiacum and released from it into the blood by a calcium-dependent mechanism. Amino acid and fast atom bombardment-mass spectroscopy analysis show that MI and MII are structurally related octapeptides representing the major secreted products of the cockroach corpus cardiacum. Both MI and MII are also present in the CNS and in the gut, indicating transmitter as well as hormonal functions in the cockroach. A survey in other species indicates MI may be present in invertebrates other than insects, but neither was found in the rat. The MI and MII peptides have clear chemical affinities to two previously described invertebrate peptides, locust adipokinetic hormone and crustacean red pigment concentrating hormone, as well as sharing biological activity with these peptides. Our results provide further evidence for the existence of a large family of structurally related peptides with divergent functions in a variety of invertebrate types.
\end{abstract}

Bioactive peptides in both vertebrates and invertebrates can be grouped into distinct families according to their biological functions and chemical structures (see Hollt, 1983, and Greenberg and Price, 1983, for reviews). Such grouping may reflect evolutionary and genetic relationships between peptide hormones, transmitters, and modulators. In recent years evidence has accumulated indicating that there exists in invertebrates a large family of structurally related but functionally diverse peptide hormones (Mordue and Stone, 1981; Greenberg and

\footnotetext{
${ }^{1}$ This work was supported by National Science Foundation Grant BNS-8202515, National Institutes of Health Grant 5T32GM-07151, and E. I. duPont de Nemours \& Co. M. S. is a National Institute of Mental Health Research Career Development Awardee (K100325). We thank Dr. K. L. Rinehart (Department of Chemistry, University of Illinois) for doing mass spectroscopy measurements, Dr. R. Heinrickson (Department of Biochemistry, University of Chicago) for performing amino acid analyses, and Mary-Kate Worden for help with some dissections. Animals were generously supplied by Johnson Wax Co. (Periplaneta americana) and Dr. D. Gibbs of DePaul University (Manduca sexta).

${ }^{2}$ To whom correspondence should be addressed.
}

Price, 1983). The existence of this family is suggested by structural and functional studies on two peptides isolated from arthropods. These are adipokinetic hormone or AKH (Stone et al., 1976) from the locust Locusta migratoria and red pigment concentrating hormone or RPCH (Carlsen et al., 1976) from the prawn Pandalus borealis. Both have similar amino acid sequences and are terminally blocked in the same way. Both can mediate the liberation of diglycerides from the locust fat body (Pines et al., 1981). Here we provide evidence for the existence of two additional members of this family in the American cockroach Periplaneta americana.

Our aim was to identify and purify peptides which caused contraction of insect skeletal muscle. The insect gut peptide proctolin (Brown and Starratt, 1975; Starratt and Brown, 1975) was recently shown to be a co-transmitter with L-glutamate at the neuromuscular junction for a subpopulation of cockroach skeletal motoneurons (O'Shea and Bishop, 1982; Adams and O'Shea, 1983). Therefore, the present study was undertaken as a prelude to finding further examples of peptides active in neuromuscular transmission. We examined the corpus cardiacum, a neurosecretory organ of Periplaneta americana, because, although no peptides have been completely 
characterized from it, it is a rich source of myoactive factors (Raabe, 1982). The corpus cardiacum of the locust was also the source of adipokinetic hormone purified by Stone et al. (1976). The corpora cardiaca are the most prominent organs in the insect neurosecretory system (Cazal, 1948). They are paired, lobed structures that lie behind the brain to which they are connected by three nerves. The corpus cardiacum contains intrinsic glandular cells and also receives terminals from neurosecretory cells that project axons from the brain (Mason, 1973). Therefore, it is a potential site of release for hormones synthesized locally and for hormones synthesized in the central nervous system (CNS). Hormones released from the organ enter the circulatory system (Orchard et al., 1981).

We have purified and characterized two myoactive peptides (MI and MII) from the corpora cardiaca of the cockroach that have clear similarities of structure and bioactivities to locust AKH and prawn RPCH. Here we describe the purification, identify a site of synthesis, demonstrate calcium-dependent release, describe three bioactivities, and establish the amino acid compositions and molecular weights of both. We also show that these peptides are not restricted to the corpus cardiacum but are present in the CNS and gut. This suggests transmitter as well as hormonal functions. Evidence is provided for close structural homologies with AKH, RPCH, and two other insect peptides which have been partially chemically characterized. These are AKH II (Carlsen et al., 1979) and neurohormone D (Bauman and Gersch, 1982). A limited survey of presence in other species indicates a distribution of these compounds beyond insects.

\section{Materials and Methods}

Animals. Approximately 3000 adult specimens of the American cockroach $P$. americana were used in this study and were the source of the peptides. The paired lobes of the corpora cardiaca were removed by dissecting the organ from the heads of $\mathrm{CO}_{2}$-anesthetized animals. Specimens of the locust Schistocerca nitens, from our own laboratory culture, were used for the blood lipid and muscle bioassays. In addition, the following organisms were used to investigate the interspecies distribution of the cockroach peptides: the moth Manduca sexta, the locust $S$. nitens, the crayfish Procambarus clarkii, and the albino laboratory rat.

Bioassays. Tests for three types of bioacitivy-(1) contraction of skeletal muscle, (2) effects on the beating frequency of an accessory heart muscle, and (3) elevation of blood lipid-were carried out in $S$. nitens.

The first two activities were measured simultaneously by monitoring the movements of a skeletal muscle, the extensor tibialis muscle of the hindleg. This muscle performs two functions. It extends the tibia when excited by motoneurons, and in the absence of neural stimulation it possesses a myogenic heart-like rhythm of contraction and relaxation which is thought to aid in the movement of blood and air into distal regions of the leg (Hoyle and O'Shea, 1974; Evans and O'Shea, 1978). The heart-like properties of the extensor muscle are limited to a small proximal bundle of fibers. To perform the assay the hindleg was removed, the femur was immobilized, and the movements of the tibia were monitored by a photoelectric diode and a Brush 220 pen recorder. The myogenic muscle fibers were exposed by cutting a window into the proximal cuticle of the femur. Samples were dissolved in a physiological saline $(140 \mathrm{~mm} \mathrm{NaCl}, 5 \mathrm{mM}$ $\mathrm{KCl}, 5 \mathrm{mM} \mathrm{CaCl}, 1 \mathrm{mM} \mathrm{MgCl}, 4 \mathrm{mM} \mathrm{NaHCO}, 5 \mathrm{mM}$ trehalose, $90 \mathrm{~mm}$ sucrose, $5 \mathrm{mM}$ HEPES, $\mathrm{pH} \mathrm{7.2)} \mathrm{and}$ applied in 1- $\mu$ l aliquots through the window directly onto the myogenic fibers of the extensor muscle. Between sample presentations the muscle was washed with $1 \mathrm{ml}$ of the physiological saline.

The blood lipid assay was performed with the BioDynamics/bmc Total Lipids Assay Kit (Catalogue Number 124303). The protocol used followed closely that described by Stone and Mordue (1980). Samples were assayed by dissolving two corpora cardiaca equivalents of high pressure liquid chromatography (HPLC)-purified peptide in $20 \mu \mathrm{l}$ of physiological saline. This volume was injected into the circulatory system through the intersegmental cuticle of the abdomen. Control animals were injected with $20 \mu \mathrm{l}$ of saline. Prior to injection, a $5-\mu \mathrm{l}$ sample of blood (hemolymph) was removed. One hour after sample injection, a second 5- $\mu$ l sample was taken. Lipid content was determined by heating the blood samples in sulfuric acid and measuring the absorbance at $535 \mathrm{~nm}$ produced upon addition of vanillin and phosphoric acid. A calibration curve was established using standards of cholesterol. One adipokinetic activity unit is defined as $1 \mu \mathrm{g}$ of lipid/ $\mu \mathrm{l}$ of hemolymph in $1 \mathrm{~h}$.

Extraction and purification. The glandular lobes of the corpora cardiaca were removed under physiological saline and transferred to a plastic vial containing $200 \mu \mathrm{l}$ of a mixture of methanol, water, and acetic acid (90:9:1). Up to 400 corpora cardiaca were accumulated in this volume prior to being homogenized by microprobe sonication (Branson 200). The extraction medium was kept at $-70^{\circ} \mathrm{C}$ while accumulating the glands. Following sonication for $5 \mathrm{~min}$ the vial was centrifuged at $9000 \times \mathrm{g}$ for $5 \mathrm{~min}$ and the supernatant removed and dried under reduced pressure at $60^{\circ} \mathrm{C}$. Extracts of other tissues were prepared in a similar way and were also subjected to an additional purification prior to being analyzed by HPLC. This involved redissolving the dry extract in $1 \mathrm{ml}$ of water and loading it onto a small cartridge filled with $\mathrm{C}_{18}$ reverse-phase packing (Sep-Pak, Waters Associates). The cartridge was flushed with $15 \%$ methanol-water and then with $5 \mathrm{ml}$ of $80 \%$ methanol, which was collected and dried. Samples prepared in this way were redissolved prior to HPLC in either water, $1 \mathrm{~mm}$ ammonium acetate ( $\mathrm{pH} 4.5$ ), or $10 \mathrm{mM}$ sodium phosphate (pH 6.9). The solvent selected depended on the specific HPLC eluting buffer being used. The HPLC columns used were analytical scale reverse-phase containing $10 \mu$ of $\mathrm{C}_{18}$ packing (Waters $\mu$-Bondapak). Eluting buffers consisted of either water, $1 \mathrm{mM}$ ammonium acetate $(\mathrm{pH} 4.5)$, or $10 \mathrm{mM}$ sodium phosphate ( $\mathrm{pH} 6.9)$ which were mixed with various proportions of acetonitrile by twin-pump delivery systems (Waters M45 or Altex 110A pumps). Delivery control systems (Waters Solvent Programmer 660 and Altex 440 Controller) were used to deliver the solvent 
isocratically or with a linearly increasing gradient of acetonitrile. The flow rate was $1 \mathrm{ml} / \mathrm{min}$ (Waters system) or $1.5 \mathrm{ml} / \mathrm{min}$ (Altex system). Compounds eluted from the column were detected by their optical density at 214 nm (Waters 440 Absorbance Detector) and by their fluorescence at $276 \mathrm{~nm}$ excitation (Schoeffel, FS 970). The limit of detection (barring interfering compounds eluting nearby) was approximately 1 pmole. For initial screening on the muscle biossay the efflux was collected by an automatic fraction collector (Gilson). Fractions were dried and redissolved in physiological saline prior to assaying. Large-scale purifications (200 to 400 pairs of corpus cardiacum) for amino acid analysis and fast atom bombardment (FAB)-mass spectrometry were achieved in two steps after the extraction in acid methanol. The dried extract was dissolved in $100 \mu \mathrm{l}$ of $1 \mathrm{mM}$ ammonium acetate $(\mathrm{pH} 4.5)$ and chromatographed on a 25 to $50 \%$ linear gradient ( $1 \%$ per minute) of acetonitrile. The two peaks of optical density $\left(\mathrm{OD}_{214}\right.$ ) corresponding to the biological activity were collected by hand into separate vials. These fractions were then reduced in volume by evaporation of the organic solvent at $60^{\circ} \mathrm{C}$ under reduced pressure. They were then separately rechromatographed using unbuffered water and a linear gradient of 25 to $50 \%$ acetonitrile. The compounds, now purified to apparent homogeneity, were again collected by hand into plastic vials.

In vitro synthesis and incorporation of $\left[{ }^{3} H\right]$ tryptophan. The glandular lobes ( 3 to 10 pairs) of the corpora cardiaca were incubated sealed under oxygen for $18 \mathrm{hr}$ at room temperature in $100 \mu \mathrm{l}$ of physiological saline containing $10 \mu \mathrm{Ci}$ of $\left[\mathrm{L}-5-{ }^{3} \mathrm{H}\right]$ tryptophan $(27 \mathrm{Ci} / \mathrm{mmol}$, AmershamSearle). After incubation the glands were washed in several changes of physiological saline without $\left[{ }^{3} \mathrm{H}\right]$ tryptophan. They were then homogenized in the acidic methanol mixture, and after centrifugation the supernatant was dried under reduced presure at $60^{\circ} \mathrm{C}$. The dried sample was dissolved in $10 \mathrm{~mm}$ sodium phosphate $\mathrm{pH}$ 6.9) and fractionated by reverse-phase HPLC. Fluorescent compounds in the efflux from the column were detected by exciting at $276 \mathrm{~nm}$. The efflux was collected by an automated fraction collector. The radioactivities of the ${ }^{3} \mathrm{H}$ isotope were measured in individual fractions (size $0.75 \mathrm{ml}$ ) by liquid scintillation counting. The profile of counts was compared to the chromatographic record of the fluorescence.

Potassium-stimulated calcium-dependent release. The ability of the corpora cardiaca to release the peptides was investigated by perfusing the glands with physiological saline and with saline of altered ionic composition. In order to concentrate hydrophobic compounds being released into the perfusate, the glands were placed on a steel screen in a small chamber (Swinny Filter Holder, Millipore) which was attached above a cartridge containing reverse-phase packing $\left(\mathrm{C}_{18}\right.$ Sep-Pak, Waters Associates). The perfusate was drawn from a reservoir above the chamber by a peristaltic pump. Further details of this method have been described (Adams and O'Shea, 1983).

Up to 30 corpora cardiaca were perfused in succession with $10 \mathrm{ml}$ of (1) physiological saline (see above); (2) saline in which the potasium concentration was elevated to $50 \mathrm{~mm}$, replacing an equimolar concentration of sodium and to which calcium was not added but was replaced by magnesium; (3) elevated potasium saline with physiological levels of calcium ( $5 \mathrm{~mm}$ ) and magnesium (1 mM). Hydrophobic compounds contained in each of the three perfusates were trapped on the $\mathrm{C}_{18}$ of the Sep-Pak cartridge. A separate cartridge was used for each saline condition. Salts and sugars were removed from each cartridge by flushing with $2 \mathrm{ml}$ of water. Peptides were then eluted with $3 \mathrm{ml}$ of methanol. The methanol was evaporated, and the dried sample was redissolved in $50 \mu \mathrm{l}$ of $10 \mathrm{~mm}$ sodium phosphate $(\mathrm{pH} 6.9)$ prior to being analyzed on an analytical $\mathrm{C}_{18}$ column using fluorescence at $276 \mathrm{~nm}$ to detect the presence of tryptophan-containing peptides in the column efflux. At the end of the experiment the corpora cardiaca were removed from the chamber, blotted, and homogenized in the acid methanol extraction mixture. The extract of the glands was prepared for HPLC purification and quantification of the levels of the peptides remaining after release. These levels were compared to the amounts released and extracted from the perfusates by diluting the corpora cardiaca extract to produce fluorescence peaks similar to those detected in the superfusates. The dilution factor provided an estimate of the amount released relative to the total in that particular experiment.

Amino acid composition and molecular weight determination. Following double HPLC purification using two different gradient solvent systems (1 $\mathrm{mM}$ ammonium acetate $(\mathrm{pH} 4.5)$ and a 25 to $50 \%$ linear gradient of acetonitrile followed by water and the 25 to $50 \%$ gradient), samples of MI and MII were collected into acidwashed Pyrex tubes. The solvents were flash evaporated. Samples were covered with distilled $5.7 \mathrm{M} \mathrm{HCl}$, and $1 \mathrm{mg}$ of phenol was added. The tubes were sealed in vacuo $(20$ $\mu$ Torr) and incubated at $110^{\circ} \mathrm{C}$ for 22 or $27 \mathrm{hr}$. Peptides extracted from 200 to 400 corpora cardiaca were quantitatively detected using an automated amino acid analyzer (Dyonics, model D502). A control chromatograph of a water sample was fractionated under identical conditions, and blank fractions corresponding to the elution time of MI and MII were collected and analyzed. The amino acid levels appearing in this control were subtracted from sample values. All corrections were less than $0.5 \mathrm{nmol}$.

Molecular weights were determined by fast atom bombardment mass spectroscopy (FAB-MS) carried out on a VG Analytical FAB mass spectroscope. Xenon was the bombarding gas and glycerol was the dispersant matrix. A description of this technology has been published recently (Rinehart, 1982).

Distribution studies. A limited survey, using HPLC purification, of the distribution of the two peptides in $P$. americana and in species other than $P$. americana was undertaken. In general, the same purification procedure was used in this survey as described above. The additional extract clean-up step using a $\mathrm{C}_{18}$ Sep-Pak was required prior to HPLC (see above). Co-migration with MI and MII of peaks detected by absorbance $(214 \mathrm{~nm})$ of fluorescence $(276 \mathrm{~nm})$ was the criterion used for a positive identification. Levels of putative $\mathrm{MI}$ and $\mathrm{MII}$ in the survey are expressed as picomoles per organ. 


\section{Results}

Identification of two myoactive factors. A pooled extract of the corpora cardiaca of male and female $P$. americana causes contraction of the main extensor muscle of the hindleg of $S$. nitens. Part of this muscle is specialized as an accessory heart and produces a myogenic rhythm of contraction. The corpora cardiaca extract also stimulates this rhythm, producing a marked acceleration of the frequency of contraction and relaxation. When the corpora cardiaca extract is fractionated by reverse-phase HPLC, the activities of the crude extract are largely accounted for by two fractions of bioactivity corresponding to two major peaks of absorbance at $214 \mathrm{~nm}$ (Fig. 1). Because of their myoactivity these have been called MI and MII. The separate extracts from male and female animals contain comparable levels of both MI and MII.

The responses shown in Figure 1 represent activity of the HLPC-fractionated MI and MII. Assays were performed using 0.002 of a single corpus cardiacum, concentrated into $1 \mu \mathrm{l}$ of saline for application to the muscle. Both produce a contraction of the extensor muscle (upward movement of the lower trace) and an increase in the beating frequency of the accessory heart. MI and MII responses cannot be distinguished qualitatively, suggesting that MI and MII activity may be produced by similar compounds. However, when the same corpora cardiaca equivalents are presented, the muscle appears to be more sensitive to MI than MII (Fig. 1). This apparent difference in the potency of MI and MII in this assay is correlated with the consistently higher levels of $214 \mathrm{~nm}$ absorbance associated with MI. The optical density ratio (MI:MII) is typically about $3: 1(\mathrm{X}=2.9 \pm 0.43 \mathrm{SD})$. The MI activity is associated with $0.0034 \pm 0.001 \mathrm{OD}_{214}-\mathrm{ml}$ per corpus cardiacum, and MII is associated with 0.00117 $\pm 0.0004 \mathrm{OD}_{214}-\mathrm{ml}$ per corpus cardiacum. This consistent observation suggested that the relative abundance of the compounds associated with MI and MII may be about 3:1 also. The amino acid analysis confirmed this (see below). Using the values of $46 \mathrm{pmol}$ of $\mathrm{MI}$ and $15 \mathrm{pmol}$ MII per corpus cardiacum we have estimated threshold sensitivity in the muscle biossay of about $10^{-8}$ to $5 \times$ $10^{-9} \mathrm{M}$ for both factors. In the example illustrated (Fig. 1) the OD peak associated with MI has been divided roughly equally into fractions 3 and 4, with a small tail into fraction 5 . The second peak, associated with MII activity, falls almost precisely over fraction 11 , with a leading edge in 10. The bioactivity closely parallels this fractionation. Thus, prominent bioactivity is seen in 3 , 4 , and 11, with weak heart acceleration and contraction seen in fractions 5 and 10. Additional evidence for the relative abundance, chemical nature, and similarity of MI and MII was provided by detecting simultaneously the absorbance at $214 \mathrm{~nm}$ and the fluorescence by exciting at $276 \mathrm{~nm}$. Both MI and MII absorbance peaks are also fluorescent (Fig. 2, $E$ and $E^{\prime}$ ), suggesting the presence of either tyrosine or tryptophan. The fluorescence associated with the absorbance peaks provided an additional distinguishing characteristic, and this is used in experiments described below as a criterion of identification of MI and MII.

The correlation between the relative peak heights and the relative potencies of MI and MII, coupled with the co-elution of the bioactivity with the detected peaks in different solvent systems, strongly suggested that the compounds producing the absorbance and fluorescence peaks are responsible for the bioactivity of MI and MII. With this as a fundamental assumption, we have proceeded to characterize the chemical nature of the MI and MII absorbance peaks.

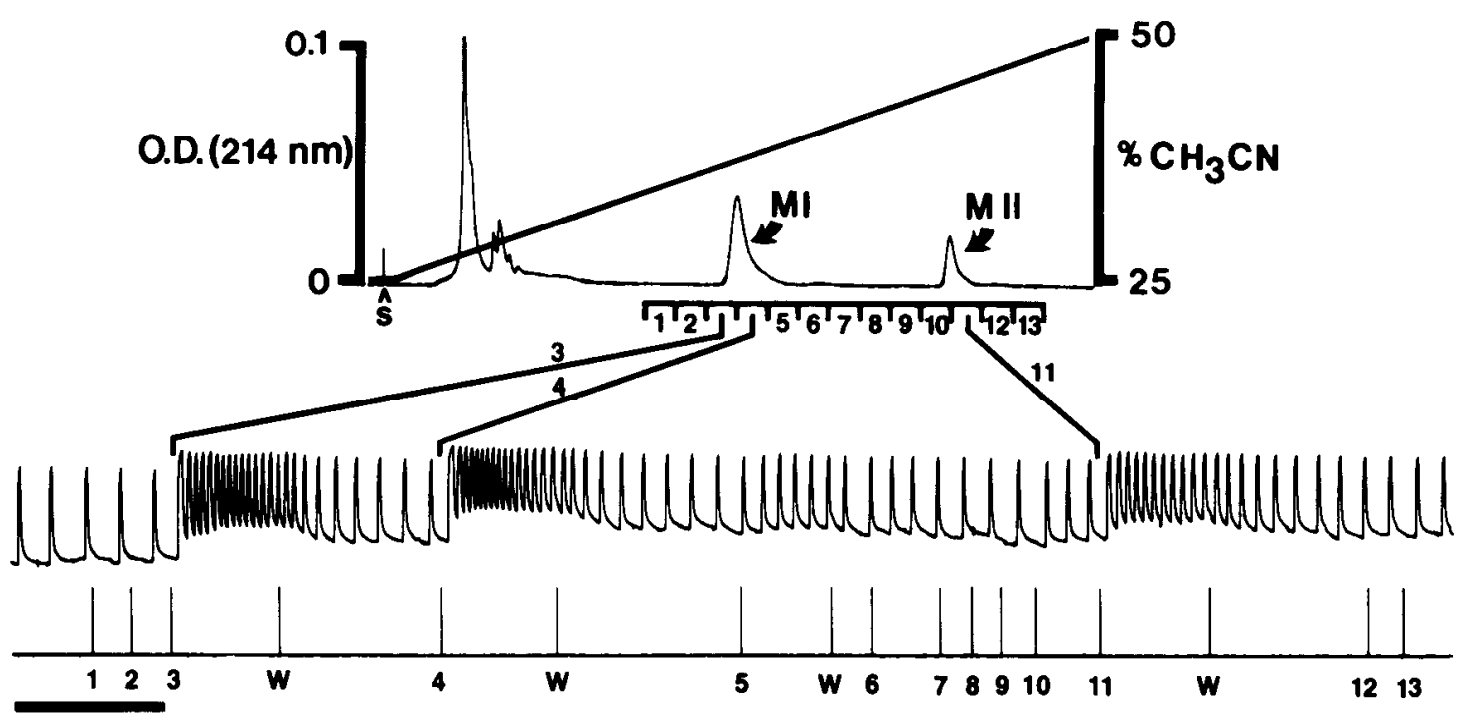

Figure 1. Identification of two myoactive factors (MI and MII) in an HPLC fractionation of an extract of 10 corpora cardiaca. The upper part shows the chromatographic record; $S$ indicates the start. Eluting buffer was $1 \mathrm{~mm}$ ammonium acetate (pH 4.5 ) and a linear gradient (gradient indicated) of between $25 \%$ and $50 \%$ acetonitrile. Fractions numbered 1 to 1.3 (1-ml fractions) were collected and bioassayed (lower part of figure). The numbers under the lowest trace indicate when that fraction was applied. The $W$ indicates saline wash. The monitor of muscle movement (contraction is an upward deflection) shows that fractions 3 , 4 , and 11 are most active and cause an increase in beating frequency and a tonic muscle contraction. Time scale (horizontal bar) is $5 \mathrm{~min}$. 
In summary, our search for myoactive factors established the presence in the corpus cardiacum of two similar bioactivities, each apparently abundant, easily detected, and relatively hydrophobic compounds that may contain tyrosine or tryptophan. Establishing that a compound is bioactive is, of course, not sufficient by itself to conclude that it has a biological role as a hormone or transmitter. Therefore, to confirm that MI and MII were of biological significance to the organism, we attempted to establish that they could be released from the corpus cardiacum in a calcium-dependent manner.

Calcium-dependent release of $M I$ and MII. Isolated corpora cardiaca were superfused at $1 \mathrm{ml} / \mathrm{min}$ with 10 $\mathrm{ml}$ of three saline solutions. The first contained physiological levels of salts, the second contained elevated potassium and zero calcium, and the third contained elevated potassium with normal calcium (see "Materials and Methods"). The $10 \mathrm{ml}$ of each solution, after they passed over the corpora cardiaca, were passed through a separate disposable column containing activated $\mathrm{C}_{18}$ reverse-phase packing (Sep-Pak, Waters Associates). The compounds retained from the saline solutions by each column were separated on an analytical $\mathrm{C}_{18}$ column and detected by $214 \mathrm{~nm}$ absorbance and $276 \mathrm{~nm}$ fluorescence. An example of such an experiment is shown in Figure 2.

Our results show that when the corpus cardiacum is exposed to saline containing calcium and elevated levels of potassium, compounds co-migrating with MI and MII and sharing their characteristic ratio of absorbance and fluorescence appear in the superfusate. When the corpora cardiaca are superfused with either physiological saline or with elevated potassium saline without calcium, MI and MII cannot be detected. We interpret this result to mean that a 10-fold increase in potassium concentration induces a depolarization of MI-containing and MII-containing cells in the corpus cardiacum, and in the presence of external calcium this depolarization leads to the secretion of MI and MII. In the organism this would produce a release of MI and MII directly from the corpus cardiacum into the blood or hemolymph. The evidence that the released compounds are MI and MII is as follows. First, both $214 \mathrm{~nm}$ absorbance and $276 \mathrm{~nm}$ fluorescence peaks (Fig. 2, $D$ and $D^{\prime}$ ) co-elute exactly with standards of MI and MII (Fig. 2, $A$ and $A^{\prime}$ ). Second, the relative absorbance and fluorescence for each peak are similar in both extracts and released experiments. Finally, when the compounds isolated and purified from the superfusate are applied to the muscle bioassay, they show MI- and MII-like activity. Our release experiment results suggest that MI and MII are released in levels proportional to their abundance and that the release is in the range of 1 to $10 \%$ of the total store. In the experiment illustrated, for example, the relative amplitudes $\left(D\right.$ and $\left.D^{\prime}\right)$ of the released MI and MII are approximately equal to their relative amplitudes in the extract of the corpora cardiaca made after the experiment $\left(E\right.$ and $\left.E^{\prime}\right)$. In this experiment, taking measurements from the larger MI peak, we estimate a release of about $5 \%$ of the total available in the glands over a $10-\mathrm{min}$ exposure to the elevated potassium saline. Thus, the amplitude of MI in $1 \%$ of the extract (Fig. $2 E$ ) approximately matches that seen in a one-fifth aliquot of the release superfusate (Fig. $2 D$ ).

\section{ABSORBANCE}

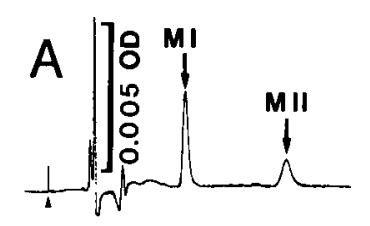

FLUORESCENCE
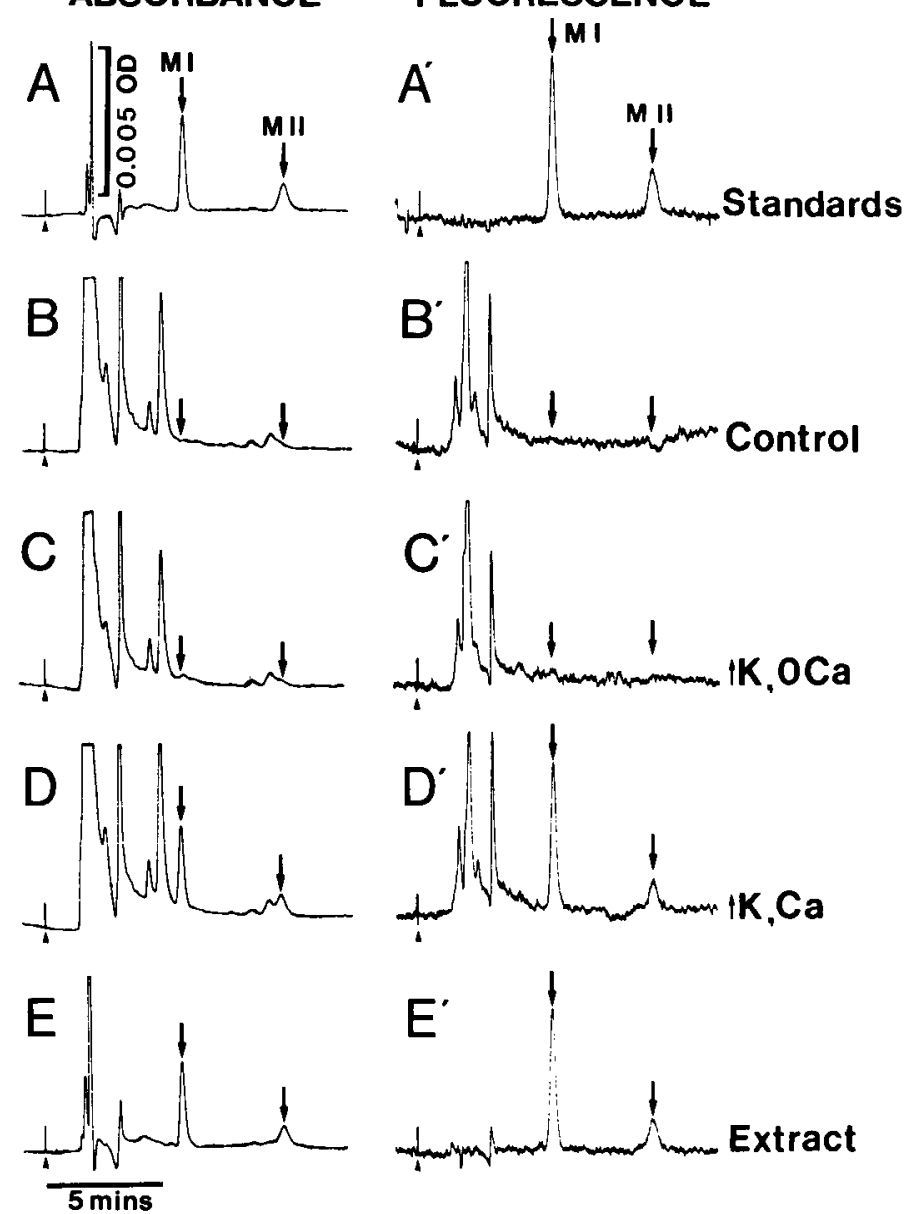

Figure 2. Chromatographic evidence for calcium-dependent release of MI and MII from 22 corpora cardiaca superfused with control and altered saline. The samples were run in 10 $\mathrm{mM} \mathrm{NaPO}_{4}, \mathrm{pH} 6.9,28 \%$ acetonitrile at a flow rate of $1.5 \mathrm{ml} /$ min. Simultaneous recording of absorbance at $214 \mathrm{~nm}(A)$ and fluorescence at $276 \mathrm{~nm}$ excitation $\left(A^{\prime}\right)$ is shown for standards of MI and MII previously HPLC purified from a corpus cadiacum extract. Arrows indicate the points where these standards would appear in the other chromatographic records. The records $B, B^{\prime}$ to $D, D^{\prime}$ show the records of a one-fifth portion of the compounds released into the superfusate (type indicated on the right). In control or physiological saline $\left(B\right.$ and $\left.B^{\prime}\right)$ or in high potassium saline without calcium ( $C$ and $C^{\prime}$ ), MI and MII are not detected. Compounds corresponding precisely to $\mathrm{MI}$ and MII are seen in $D$ and $D^{\prime}$ (high potassium with calcium). Peaks eluting early in records $B, B^{\prime}$ to $D, D^{\prime}$ are not associated with the corpora cardiaca (see $E$ and $E^{\prime}$ ) and are not significantly altered in the different saline conditions. These compounds are present in the $10 \mathrm{ml}$ of saline used in each part of the experiment and were adsorbed onto the $\mathrm{C}_{18}$ Sep-Paks. After the experiment the glands were homogenized and an HPLC record of a 0.01 portion of the extract is shown in $E$ and $E^{\prime}$. Records of MI and MII peaks in $E, E^{\prime}$ and D, D' are approximately equal, indicating a release of about $5 \%$ of both peptides in these experiments $\left(D, D^{\prime}\right.$ is a one-fifth portion of the release superfusate).

Amino acid compositions and molecular weights. The two peaks corresponding to MI and MII were purified from 200 to 400 corpora cardiaca in two steps of reversephase HPLC (see "Materials and Methods") to produce compounds of apparent homogeneity (Fig. 3). The indi- 
vidual MI and MII compounds were collected for either amino acid analysis or FAB-mass spectrometry.

The results of amino acid analysis are shown in Table I. Due to fluorescence at $276 \mathrm{~nm}$, we assume the presence of either tyrosine or tryptophan in both MI and MII. Tyrosine was not detected by our amino acid analysis; therefore, we infer by elimination the presence of tryptophan, which is destroyed during acid hydrolysis. The presence of tryptophan is confirmed in MI and MII by $\left[{ }^{3} \mathrm{H}\right]$ tryptophan incorporation in in vitro synthesis experiments described below and by the molecular weight determinations.

Molecular weights, as determined by FAB-mass spectroscopy, are 972 for MI and 987 for MII. These measurements resolve the ambiguities inherent in the amino

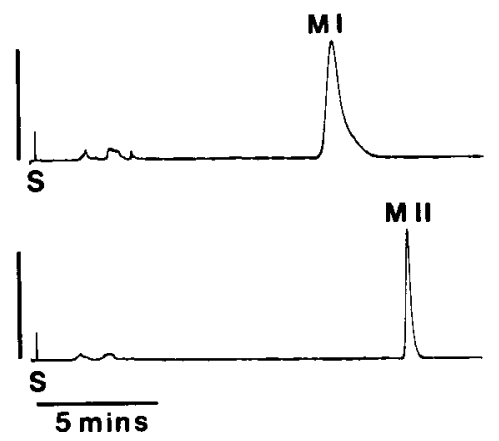

Figure 3. Purification to homogenity of MI and MII from 400 corpora cardiaca in two steps of reverse-phase HPLC. From an initial HPLC purification of the crude methanol extract (see, for example, Fig. 1) the MI and MII peaks were collected and then individually HPLC purified using unbuffered water and a linear gradient of from $25 \%$ to $50 \%$ acetonitrile. Optical density profiles at $214 \mathrm{~nm}$ of these second steps of HPLC purification are shown here. The vertical scale bar is 1 OD unit. When the peak OD measurements (1.1 OD for MI and 1.3 OD for MII) are integrated, the relative abundances are $1.21 \mathrm{OD}$ $\mathrm{ml} \mathrm{MI}$ and $0.39 \mathrm{OD}$-ml MII or about $0.0003 \mathrm{OD}$ and $0.0001 \mathrm{OD}$ per gland.

TABLE I

Results of amino acid analysis of MI and MII

\begin{tabular}{|c|c|c|c|c|c|c|}
\hline $\begin{array}{c}\text { Detected } \\
\text { Amino Acid }\end{array}$ & MI & $\begin{array}{c}\text { MI } \\
\text { Ratio }\end{array}$ & $\begin{array}{c}\text { MI } \\
\text { Composition }\end{array}$ & MII & $\begin{array}{c}\text { MII } \\
\text { Ratio }\end{array}$ & $\begin{array}{c}\text { MII } \\
\text { Composition }\end{array}$ \\
\hline & nmol & & & nmol & & \\
\hline Asx (Asn or Asp) & 21.6 & 1.84 & 2 & 3.59 & 0.93 & 1 \\
\hline Thr & $\mathrm{ND}^{\alpha}$ & & & 5.74 & 1.48 & 2 \\
\hline Ser & 7.5 & 0.64 & 1 & ND & & \\
\hline $\begin{array}{l}\text { Glx (Glu, Gln or } \\
\text { PGlu) }\end{array}$ & 11.7 & 1 & 1 & 3.89 & 1 & 1 \\
\hline Pro & 13.5 & 1.13 & 1 & 3.98 & 1.03 & 1 \\
\hline Gly & 0.5 & 0.05 & & 0.34 & 0.08 & \\
\hline Ala & 0.4 & 0.03 & & 0.34 & 0.08 & \\
\hline Val & 10.2 & 0.87 & 1 & ND & & \\
\hline Ilu & 0.6 & 0.05 & & ND & & \\
\hline Leu & 0.7 & 0.06 & & 3.88 & 1.00 & 1 \\
\hline Try & 0.3 & 0.02 & & ND & & \\
\hline Phe & 10.6 & 0.9 & 1 & 3.25 & 0.84 & 1 \\
\hline His & 0.3 & 0.02 & & 0.18 & 0.05 & \\
\hline Lys & 0.1 & 0.01 & & 0.12 & 0.03 & \\
\hline Trp & ND & & & 0.44 & 0.11 & \\
\hline Arg & 0.7 & 0.06 & & ND & & \\
\hline
\end{tabular}

${ }^{a} \mathrm{ND}$, not detected. acid analysis and are exactly consistent with the following. First, each peptide contains a single tryptophan residue. Second, for each there is a pGlu at the amino terminal and the carboxy terminals are amidated. Finally each Asx is resolved to be asparagine.

The amino acid analysis allowed us to establish relative and absolute abundances of MI and MII in the corpus cardiacum. Table I shows that the consistently observed optical density ratio of about 3:1 (see above) is reflected in the ratios of abundance. For example, in the data illustrated in Table I, assuming one Glx per molecule, there are $11.7 \mathrm{nmol}$ in MI and $3.89 \mathrm{nmol}$ in MII, a ratio of 3:1. These quantities of MI and MII were obtained from 250 corpora cardiaca, so the average abundances per gland are about $46 \mathrm{pmol}$ of MI and about $15 \mathrm{pmol}$ of MII. Using these values, we estimate that the muscle responses illustrated in Figure 1 are produced by concentrations of $5 \times 10^{-8} \mathrm{M} \mathrm{MI}$ (fractions 3 and 4) and about $3 \times 10^{-8}$ M MII (fraction 11).

Further characterization of the structures of $\mathrm{MI}$ and MII by FAB-mass spectroscopy is currently being pursued.

Site of synthesis and confirmation of tryptophan presence. Peptides in the corpus cardiacum may be synthesized locally by intrinsic glandular cells or transported there in nerves from sites of synthesis in the CNS. To investigate whether the MI and MII peptides are synthesized locally, we cultured isolated glands in vitro for up to $20 \mathrm{hr}$ in physiological saline containing $\left[{ }^{3} \mathrm{H}\right]$ tryptophan. An isotope of tryptophan was selected for this experiment because its incorporation into MI and MII would both establish the ability of the corpus cardiacum to synthesize the peptides and at the same time directly confirm the presence of tryptophan in both.

The results of an 18-hr incubation of three corpora cardiaca with $10 \mu \mathrm{Ci}$ of $\left[{ }^{3} \mathrm{H}\right] \mathrm{Trp}$ are shown in Figure 4 . After the incubation the corpora cardiaca were washed, extracted, and purified by reverse-phase HPLC. Co-migrating with the MI and MII peaks are two tritium peaks of the same relative amplitudes. We interpret the results of in vitro incorporation of tritium into $\mathrm{MI}$ and $\mathrm{MII}$ in this experiment to show that (1) both peptides contain tryptophan, (2) the corpus cardiacum is capable of synthesizing MI and MII, and (3) the peptides appear to be synthesized at rates directly related to their relative stored levels. The experiment does not exclude the possibility of other sites of synthesis for MI and MII. In fact, results of a distribution survey described below suggest that these peptides are synthesized in other structures.

Distribution within the cockroach and in other species. A limited survey, using HPLC isolation, of the distribution of MI and MII in the cockroach CNS and gut and their presence in other species has been undertaken. Results of their distribution in cockroach are shown in Table II. Results of their possible presence in other species are shown in Table III.

AKH-like and RPCH-like bioactivity of $M I$ and $M I I$. The well established correlation of structure and function for AKH and RPCH and their chemical similarity to MI and MII, indicated by amino acid analysis, suggested that these previously described peptides might afford clues to the amino acid sequences of MI and MII. 


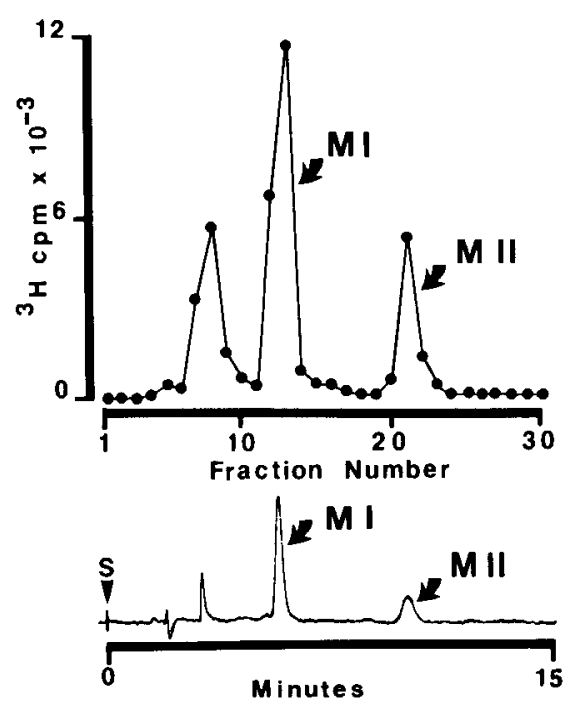

Figure 4. Incorporation of $\left[{ }^{3} \mathrm{H}\right]$ tryptophan into $\mathrm{MI}$ and MII by corpus cardiacum. Three glands were incubated for $18 \mathrm{hr}$ with $10 \mu \mathrm{Ci}$ of $\left[{ }^{3} \mathrm{H}\right] \mathrm{Trp}$. The glands were extracted as usual and the extract chromatographed isocratically as in Figure 2. The lower part of the figure shows the fluorescence profile of this run $(S=$ start). The upper trace shows the radioactivity contained in $0.75-\mathrm{ml}$ fractions corresponding to the fluorescence profile below. The fluorescence peaks of MI and MII each correspond to major tritium peaks. The first tritum peak is probably comprised primarily of free $\left[{ }^{3} \mathrm{H}\right] \mathrm{Trp}$.

TABLE II

$M I$ and $M I I$ content of various cockroach organs

\begin{tabular}{lcc}
\hline & MI & MII \\
\hline Brain & $0.9^{a}$ & 0.01 \\
VNC & 0.3 & ND $(\leq 0.03)$ \\
Foregut & 0.2 & ND $(\leq 0.06)$ \\
Midgut & $\mathrm{ND}^{b}(\leq 0.4)$ & $\mathrm{ND}(\leq 0.3)$ \\
Hindgut & $\mathrm{ND}(\leq 0.2)$ & $\mathrm{ND}(\leq 0.02)$ \\
\hline
\end{tabular}

${ }^{a}$ All values are in picomoles per organ extracted.

${ }^{b} \mathrm{ND}$, not detected.

TABLE III

Results of HPLC survey of various animals for MI and MII (see the text)

\begin{tabular}{lcc}
\multicolumn{1}{c}{ Species } & MI & MII \\
\hline $\begin{array}{l}\text { S. nitens (locust) } \\
\text { Corpus cardiacum }\end{array}$ & $\mathrm{ND}^{a}(\leq 0.03)$ & $\mathrm{ND}(\leq 0.08)$ \\
Brain & $\mathrm{ND}(\leq 0.04)$ & $0.1^{b}$ \\
$\begin{array}{l}\text { Manduca sexta (moth) } \\
\text { Corpus cardiacum }\end{array}$ & $\mathrm{ND}(\leq 0.009)$ & $\mathrm{ND}(\leq 0.02)$ \\
$\quad$ Brain & 0.09 & $\mathrm{ND}(\leq 0.03)$ \\
$\begin{array}{l}\text { Procambarus clarkii (crayfish) } \\
\text { Eyestalk }\end{array}$ & 0.5 & $\mathrm{ND}(\leq 0.1)$ \\
$\begin{array}{l}\text { Post commissural organ } \\
\text { Albino rat }\end{array}$ & $\mathrm{N} . \mathrm{D} .(\leq 0.5)$ & $\mathrm{ND}(\leq 0.08)$ \\
Brain & $\mathrm{ND}(\leq 2.8)$ & $\mathrm{ND}(\leq 0.4)$ \\
Pituitary & $\mathrm{ND}(\leq 0.5)$ & $\mathrm{ND}(\leq 0.2)$ \\
\hline
\end{tabular}

${ }^{a} \mathrm{ND}$, not detected (detection limit indicated).

${ }^{b}$ All values are in picomoles per organ extracted.

With this in mind and in order to establish similarities of action among this group of peptides, we investigated the activities of MI and MII in the locust AKH bioassay.

Figure 5 shows that both MI and MII, when injected

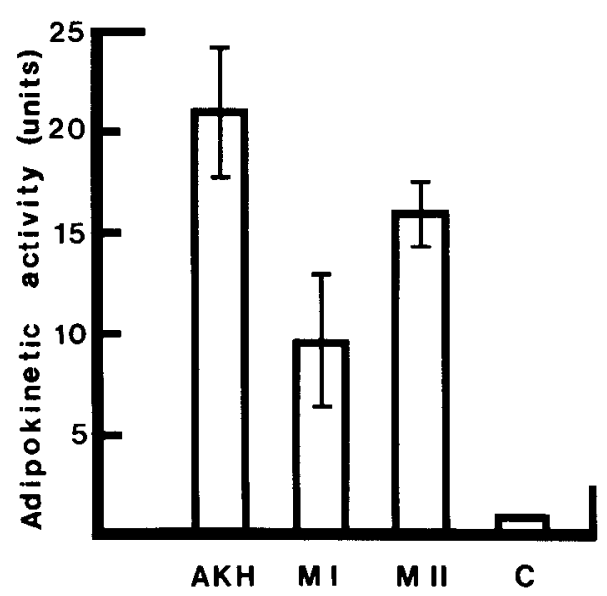

Figure 5. Adipokinetic assay of AKH, MI, MII, and saline (C). One unit of activity is $1 \mu \mathrm{g}$ of lipid/ $\mu$ l of hemolymph (blood) in $1 \mathrm{hr}$. Test animals were injected with 10 pmol of AKH $(n=4)$, about $90 \mathrm{pmol}$ of MI $(n=10)$, and about 30 pmol of MII $(n=8)$. Mean responses $\pm 1 \mathrm{SD}$ are presented.

into the locust, cause significant elevation of blood lipid levels. In this experiment, MI and MII peptides purified from two corpora cardiaca were used for each injection. Thus, the AKH-like activity was stimulated by about 90 pmol of MI and $30 \mathrm{pmol}$ of MII. The levels of stimulation are compared to the effects of $10 \mathrm{pmol}$ of $\mathrm{AKH}$ and to control injection of saline. The comparison suggests that $\mathrm{AKH}$ is about 22 times more potent per mole than MI but only 3.8 times more active than MII. This suggests that MII may be structurally more similar to AKH than is MI. The amino acid analysis also indicates this since MI has two amino acids not found in AKH (valine and serine), whereas all amino acids in MII are present in AKH (Table IV).

\section{Discussion}

By contrast to the large number of fully characterized vertebrate neuropeptides, in insects there are only two. These are proctolin, Arg-Tyr-Leu-Pro-Thr (Brown and Starratt, 1975; Starratt and Brown, 1975) and adipokinetic hormone, pGlu-Leu-Asn-Phe-Thr-Pro-Asn-TrpGly-Thr- $\mathrm{NH}_{2}$ (Stone et al., 1976). Numerous neurohormonal "factors," many of them presumed to be peptides which control development, affect rate of heart beat, and have myotropic activity and metabolic activities have, however, been described in insects. An important challenge now is the identification, purification, and chemical characterization of these factors. By analogy with other invertebrate systems in which single neurohormones, egg laying hormone, for example, have multiple functions (Chiu, et al., 1979; Strumwasser, et al., 1980), it is likely that fewer peptides exist than is suggested by the multiplicity of factors. Isolating and characterizing neuropeptides in insects will clearly improve our understanding of the neurobiology of these important organisms. We may also hope that peptides present in insects will have structural affinities with peptides in widely divergent organisms. A precedent for this was the discovery in mammals of peptides related to the molluscan peptide FMRF-NH ${ }_{2}$ (Greenberg et al., 1981; Dockray et al., 1981; 
Weber et al., 1981). In addition, because in organisms with relatively simple nervous systems we can develop model cellular preparations, the discovery of new insect peptides can potentially advance our basic understanding of how neuropeptides function on a cellular level. A precedent for this has been the characterization of proctolinergic motoneurons in $P$. americana (O'Shea et al., 1982).

We have isolated two related octapeptides with similar bioactivities that are released from and synthesized in the corpus cardiacum of $P$. americana. These peptides are of interest to us primarily for two reasons. First, they are both active on skeletal muscle, producing pronounced contraction at low concentrations. Second, they appear to be members of a growing family of structurally related but functionally diverse peptides present in arthropods (Greenberg and Price, 1983).

The meaning of the actions of MI and MII on skeletal muscle may be related to the recent finding that a subpopulation of skeletal motoneurons in $P$. americana is peptidergic (Bishop and O'Shea, 1982; O'Shea and Bishop, 1982; Adams and O'Shea, 1983). These motoneurons, which comprise about $5 \%$ of the total motor pool, are immunoreactive to antibodies against the pentapeptide proctolin. Proctolin is not structurally related to MI or MII, but its action on the insect skeletal muscle assay is similar. It accelerates the myogenic rhythm and produces a tonic contraction. Proctolin is released by an individually identified skeletal motoneuron and functions as a co-transmitter (Adams and O'Shea, 1983). The transmitter usually associated with arthropod motoneurons is L-glutamic acid, and proctolin appears to function as a skeletal muscle co-transmitter with L-glutamate in the proctolin subpopulation (Adams and O'Shea, 1983). We believe that other subpopulations of motoneurons may exist that use as co-transmitters myoactive peptides other than proctolin. Thus, it is possible that MI and MII may function in this way. Resolution of this question, however, will depend on identifying the cells which contain the MI and MII peptides that our cockroach distribution study (Table II) indicates are present in the CNS. Several pieces of evidence raise the possibility of a similar role for AKH. Recent immunocytochemical detection of neurons in the locust CNS which contain AKH or AKH-like peptides was reported (Schooneveld et al., 1983). This is the first indication that $\mathrm{AKH}$ or $\mathrm{AKH}-$ like peptides might function as transmitters as well as circulating neurohormones in the locust. In addition, $\mathrm{AKH}$ has an activity on the skeletal muscle biossay not distinguishable from the actions of MI and MII (unpublished observations). Therefore, we may speculate that, by analogy with the role of proctolin, it also may function as a co-transmitter of skeletal motoneurons. 'The possible motoneuronal identity of the $\mathrm{AKH}$-immunoreactive neurons in the locust CNS, or the possible immunological cross-reactivity with MI and MII, has not yet been established (Schooneveld et al., 1983; H. Schooneveld, personal communication).

The striking structural similarities of $\mathrm{AKH}$ and $\mathrm{RPCH}$ (Table IV) and the fact that these peptides are found in organisms phylogenetically widely separated (Insecta and Crustacea), suggests the existence in the phylum Arthropode of a large peptide family. In addition, evi- dence from their functional cross-reactivity and from structure-activity relations (Mordue and Stone, 1977, 1981) suggests that AKH and RPCH receptors are also similar. Two other peptides, compound II from the locust corpus cardiacum (Carlsen et al., 1979) and neurohormone $\mathrm{D}$ from the cockroach corpus cardiacum (Baumann and Gersch, 1982), may also be members of the AKH$\mathrm{RPCH}$ family. These peptides have been characterized by amino acid analysis which reveals a similarity of composition to AKH and RPCH. Moreover, they also share some bioactivities. For example, locust compound II has AKH-like activity in the locust blood lipid assay (Carlsen et al., 1979), and neurohormone D has cardioexcitatory action which has also been ascribed to AKH (Mordue and Stone, 1981; Baumann and Gersch, 1982). It is possible, therefore, to speculate on amino acid sequences for compound II and neurohormone D that reflect these observations, and this has been done (Greenberg and Price, 1983). Similarly, it is not unreasonable to use the known structure-activity relationships of $\mathrm{AKH}$ and $\mathrm{RPCH}$ to construct tentative sequences for MI and MII. However, the precise molecular weights determined by FAB-mass spectroscopy allow us to predict with greater confidence the presence of a pGlu $N$ terminal and an amidated carboxy terminal, consistent with the structures of $\mathrm{AKH}$ and $\mathrm{RPCH}$. The amino acids can be arranged in the sequences shown in Table IV to reflect structural homologies that are indicated by our amino acid analyses and demonstrations of AKH-like bioactivities of MI and MII. The amidated carboxy terminals of MI and MII may reflect additional homology of these peptides with AKH. In the AKH sequence, Trp is followed by Gly at its carboxy terminal. Peptide amidation has been shown in several other systems to arise from the modification of a glycine residue. For example, porcine gastrin contains a carboxy-terminal $\mathrm{Phe}-\mathrm{NH}_{2}$, and the gastrin mRNA sequence reveals the presence of Gly adjacent to the carboxy terminal Phe (Yoo et al., 1982). In the prepeptide for calcitonin a Gly follows the carboxy terminal residue of the processed peptide (Jacobs et al., 1981). Moreover, Bradbury et al. (1982) have demonstrated an enzymatic activity which catalyzes the oxidative deamination of Gly to amidate the adjacent amino acid. Therefore, we may speculate that the Trp of MI and MII may be followed by Gly in a larger precursor peptide. The presence of Gly in this position in the decapeptide $\mathrm{AKH}$ suggests a further sequence homology between AKH and the octapeptides MI, MII, and RPCH.

The peptide family now includes AKH, RPCH, compound II, neurohormone D, MI, and MII. Since the last four are not yet fully characterized peptides, we should consider whether there is evidence that they are, in fact, structurally distinct. Compound II cannot be MI because MI lacks threonine, and it cannot be MII because MII lacks serine. The reported amino acid composition of

TABLE IV

Speculative sequences of MI and MII based on likely homology with $A K H$ and RPCH (see text for justification)

MI pGlu-Val-Asn-Phe-Ser-Pro-Asn-Trp- $\mathrm{NH}_{2}$

MII pGlu-Leu-Thr-Phe-Thr-Pro-Asn-Trp- $\mathrm{NH}_{2}$

AKH pGlu-Leu-Asn-Phe-Thr-Pro-Asn-Trp-Gly-Thr-NH

$\mathrm{RPCH}$ pGlu-Leu-Asn-Phe-Ser-Pro-Gly-Trp- $\mathrm{NH}_{2}$ 
neurohormone D suggests an affinity with MI. Since neurohormone $\mathrm{D}$ did not react with dansyl chloride, the $N$-terminal amino acid is probably blocked. Moreover, the peptide is not electrophoretically mobile, indicating that aspartate probably exists as asparagine and glutamate as glutamine or pyroglutamate. The presence of tryptophan in neurohormone $\mathrm{D}$ was not determined and could not have been since it is destroyed by acid hydrolysis prior to amino acid analysis. If neurohormone $D$ does contain a single tryptophan residue, then it may be the same peptide as MI. This can only be determined by a more complete structural analysis of both peptides.

Our distribution study showing that MI may be present in crayfish and in Manduca sexta, an insect distantly related to the cockroach, suggests this peptide may be widely distributed among the arthropods. Furthermore, MII is not restricted to the cockroach but was found in the related insect $S$. nitens. However, we regard our positive findings as preliminary because the criteria of co-chromatography, while strong, is not definitive evidence of structural identity. In addition, we do not regard our negative findings to be proof of the absence of MI and MII since limited amounts of tissue were used, extraction efficiences may vary in different tissues, and in some cases complex chromatographic profiles limited sensitivity of detection. We have tried to indicate to some extent the success of our detection system in each case in Table III, but certain potential problems (extraction efficiency) remain to be explored.

\section{References}

Adams, M. E., and M. O'Shea (1983) Peptide co-transmitter at a neuromuscular junction. Science. 221: 286-289.

Bauman, E., and M. Gersch (1982) Purification and identification of neurohormone $\mathrm{D}$, a heart accelerating peptide from the corpora cardiaca of the cockroach Periplaneta americana. Insect Biochem. 12: 7-14.

Bishop, C. A., and M. O'Shea (1982) Neuropeptide proctolin (H-Arg-Tyr-Leu-Pro-Thr-OH): Immunocytochemical mapping of neurons in the central nervous system of the cockroach. J. Comp. Neurol. 207: 223-238.

Bradbury, A. F., M. D. A. Finnie, and D. G. Smyth (1982) Mechanism of C-terminal amide formation by pituitary enzymes. Nature 298: 686-688.

Brown, B. E., and A. N. Starratt (1975) Isolation of proctolin, a myotropic peptide from Periplaneta americana. J. Insect Physiol. 21: 1879-1881.

Carlsen, J., M. Christensen, and L. Josefesson (1976) Purification and chemical structure of the red pigment-concentrating hormone of the prawn Leander adspersus. Gen. Comp. Endocrinol. 30: 327-331.

Carlsen, J., W. S. Herman, M. Christensen, and L. Josefesson (1979) Characterization of a second peptide with adipokinetic and red pigment-concentrating activity from the locust corpora cardiaca. Insect Biochem. 9: 497-501.

Cazal, P. (1948) Les glandes endocrines retro-cerebrales des Insectes. Etude morphologique. Bull. Biol. Fr. Belg. Suppl. 32: $1-30$.

Chiu, A. Y., M. W. Hunkapiller, E. Heller, D. K. Stuart, L. E. Hood, and F. Strumwasser (1979) Purification and primary structure of the neuropeptide egg-laying hormone of Aplysia california. Proc. Natl. Acad. Sci. U. S. A. 76: 6656-6660.

Dockray, G. J., C. Vaillant, and R. G. Williams (1981) New vertebrate brain-gut peptide related to a molluscan neuropeptide and an opioid peptide. Nature 293: 656-657.

Evans, P. D., and M. O'Shea (1978) The identification of an octopaminergic neuron and the modulation of a myogenic rhythm in the locust. J. Exp. Biol. 73: 235-260.

Greenberg, M. J., and D. A. Price (1983) Invertebrate neuropeptides: Native and Naturalized. Annu. Rev. Physiol. 45. 271-288.

Greenberg, M. J., D. D. Painter, and D. A. Price (1981) The amide of the naturally occurring opioid Met enkephalin-Arg ${ }^{6}$. $\mathrm{Phe}^{7}$ is a potent analog of the molluscan neuropeptide FMRF amide. Neuropeptides 1: 309-317.

Hollt, V. (1983) Multiple endogenous opioid peptides. Trends Neurosci. 6: 27-31.

Hoyle, G., and M. O'Shea (1974) Intrinsic rhythmic contractions in insect skeletal muscle. J. Exp. Zool. 189: 407-412.

Jacobs, J. W., R. H. Goodman, W. W. Chin, P. C. Dee, J. F. Habener, N. H. Bell, and J. R. Potts (1981) Calcitonin messenger RNA encodes multiple polypeptides in a single precursor. Science 231: 457-459.

Mason, C. A. (1973) New features of the brain retrocerebral neuroendocrine complex of the locust Scistocerca vaga (Scudder). Z. Zellforsch. Mikrosk. Anat. 114: 19-32.

Mordue, W., and J. V. Stone (1977) Relative potencies of locust adipokinetic hormone and prawn red pigment-concentrating hormone in insect and crustacean systems. Gen. Comp. Endocrinol. 33: 103-108.

Mordue, W., and J. V. Stone (1981) Structure and function of insect peptide hormones. Insect Biochem. 11: 353-360.

Orchard, I., T. Friedel, and B. G. Loughton (1981) Release of a neurosecretory protein from the corpora cardiaca of Locusta migratoria induced by high potassium saline and compound action potentials. J. Insect Physiol. 27: 297-305.

O'Shea, M., and C. A. Bishop (1982) Neuropeptide proctolin associated with an identified skeletal motoneuron. J. Neurosci. 2: 1242-1251.

O'Shea, M., M. E. Adams, and C. A. Bishop (1982) Identification of proctolin-containing neurons. Fed. Proc. 41: 29402947.

Pines, M., A. Tietz, H. Weintraub, S. W. Applebaum, and L. Josefsson (1981) Hormonal activation of protein kinase and lipid mobilization in the locust fat body in vitro. Gen. Comp. Endocrinol. 43: 427-431.

Raabe, M. (1982) Insect Neurohormones, Plenum Press, New York.

Rinehart, K. L. (1982) Fast atom bombardment mass spectrometry. Science 218: 254-260.

Schooneveld, H., G. I. Tesser, J. A. Veenstra, and H. M. Romberg-Privee (1983) Adipokinetic hormone and AKH-like peptide demonstrated in the corpora cardiaca and nervous system of Locusta migratoria by immunohistochemistry. Cell Tissue Res. 230: 67-76.

Starratt, A. N., and B. E. Brown (1975) Structure of the pentapeptide proctolin, a proposed neurotransmitter in insects. Life Sci. 17: 1253-1256.

Stone, J. V., and W. Mordue (1980) Adipokinetic hormone. In Neurohormonal Techniques in Insects, T. A. Miller, ed., pp. 31-80, Springer-Verlag, New York.

Stone, J. V., and W. Mordue (1981) Structure and function of insect peptide hormones. Insect Biochem. 11: 353-360.

Stone, J. V., W. Mordue, K. E. Batley, and H. R. Morris (1976) Structure of locust adipokinetic hormone, a neurohormone regulates lipid utilization during flight. Nature 263: 207-211.

Strumwasser, F., L. K. Kaczmerek, A. Y. Chiu, E. Heller, K. R. Jennings, and D. P. Viele (1980) Peptides controlling behavior in Aplysia. In Peptides: Integrators of Cell and Tissue Function, F. E. Bloom, ed., pp. 197-218, Publisher, City of Publication.

Weber, E., C. J. Evans, S. Samuelsson, and J. D. Barchas (1981) Novel peptide neural system in rat brain and pituitary. Science 214: 1248-1251.

Yoo, J. O., T. C. Powell, and K. L. Agarwal (1982) Molecular cloning and nucleotide sequence of full-length $\mathrm{cDNA}$ coding for porcine gastrin. Proc. Natl. Acad. Sci. U. S. A. 79: 10491053. 